\title{
An Evaluation of Competitor Type and Size for Use in the Determination of mRNA by Competitive PCR
}

\author{
R.K. McCulloch, C.S. Choong, and D.M. Hurley
}

\author{
Department of Medicine, University of Western Australia, Research Centre, Royal Perth Hospital, Perth, W.A. 6000 Australia
}

\begin{abstract}
The technique of competitive PCR for measuring mRNA is used widely. Several variations of the method have been reported. We have evaluated some of the commonly used competitor types as part of our study into expression of the androgen receptor (AR). These included mutant, intron, deletion construct, and nonhomologous competitors, which were assessed with an emphasis on their ability to amplify the target with the same efficiency, as well as their capacity to form heteroduplexes with it. The effect of competitor size on amplification efficiency was also investigated. We found that the use of a common primer set did not guarantee equal amplification efficiencies among DNAs sharing the same primer sequences. For the competitors evaluated in this study, sequence length was the major determinant of amplification efficiency. The longest competitors were amplified with the least efficiency. Differences in amplification efficiencies were corrected for by standardizing the competitor against the target. Constructing competitors of different sizes to the target may not eliminate heteroduplex formation when they share common sequence with the target as with the intron and deletion type competitors. Such heteroduplexes may interfere with the analysis if they cannot be resolved from both the target and competitor. Use of a mutant competitor constructed by the conversion of one enzyme restriction site to another produced determinations that were independent of both heteroduplex formation and cycle number. A
\end{abstract}

method is described for generating a mutant competitor with a single PCR. The results of this study show that when competitive PCR is being used to determine absolute levels of mRNA, the competitor constructed should be assessed carefully for its ability to amplify with the same efficiency as the target and for its capacity to form heteroduplexes with it.

C mpetitive PCR for the quantitation of cellular mRNA is a rapid and highly sensitive technique. With this method, an internal standard or competitor is coamplified with the target message using a common set of primers. If the efficiency of amplification of the two species is the same, the ratio of products following PCR will reflect directly the initial amounts present enabling quantitation to be made. The competitor is distinguished from the target generally by gel electrophoresis and is usually constructed to be either of different size from the target or a mutant of it with an addition (or deletion) of a restriction site to enable size discrimination after digestion. The different sized competitor may be prepared by the inclusion of an intron to the target sequence ${ }^{(1)}$ or by the deletion of bases if suitable restriction enzyme sites are available. ${ }^{(2)}$ These strategies will result in the target and competitor sharing many common nucleotides that aim to minimize differences in amplification efficiencies between the two. Alternatively, the internal standard may be synthesized from nonhomologous bases except for the shared primer sequences. ${ }^{(3-5)}$
Initially with the mutant competitor technique, (1) either the target or the standard contained a unique restriction site that allowed differentiation of the two following digestion with a single enzyme. A refinement of this method when constructing the competitor is to replace one restriction site of the target with another and then to digest the amplified mixture with either enzyme (double-cut method). ${ }^{(6)}$

Each of the above methods has potential advantages and disadvantages. Even small differences in amplification efficiencies that might arise from using nonhomologous templates can translate into significant variation in product yields over 30 or 40 PCR cycles. When using a mutant as an internal standard, amplification efficiencies are the same but digestion-resistant heteroduplexes arise as the reaction approaches the plateau phase and may result in the equivalence point becoming cycle dependent. The ideal competitor should be readily prepared, easily distinguishable from the target, amplify with equal efficiency, provide a result that is independent of cycle number, and not require radioisotopes for quantitation.

As part of our study into androgen resistance, we needed to quantify gene expression of the androgen receptor and choose the technique of competitive PCR to achieve this. Our initial selection of competitor type, an intron competitor, yielded a construct that was not suitable for absolute quantitation of mRNA. Subsequently, we evaluated some of the other commonly used competitor types and have made some findings that may be of interest to those planning to use this technique. These findings include a 
simplified method for preparing mutant competitors that requires only a single PCR. We also noted that standards prepared using the "deletion construct" or "intron" methods may form heteroduplexes with the target, that amplifying different templates using a common primer set does not guarantee equal amplification efficiencies, and that using a mutant competitor in the double-cut mode provides determinations that are independent of heteroduplex formation. We have discussed strategies to compensate for some of the above findings and to enable all competitors, except the intron competitor, when used in the determination of the same set of samples to give very similar, reproducible results.

\section{MATERIALS AND METHODS}

Synthetic nucleotides were prepared using a Milligen/Biosearch Cyclone Plus DNA synthesizer and were diluted to a working concentration of $25 \mu \mathrm{M}$. Taq polymerase, dNTPs, and PCR buffer were supplied by Perkin-Elmer Cetus (Norwalk, CT); AMV reverse transcriptase, RNasin, and pGem-T vector were supplied by Promega (Sydney, Australia); and T4 DNA ligase was supplied by Boehringer Mannheim (Indianapolis, IN). Except for the intron competitor, which was amplified from genomic DNA, all constructs and the inserts for the nonhomologous competitor were derived from the CMVhAR3.1 plasmid, which contains the code for the androgen receptor (kindly provided by W. Tilley). ${ }^{(7)}$

\section{PCR Conditions}

The following conditions were used for PCR unless otherwise stated. Buffer composed of $10 \mathrm{~mm}$ Tris- $\mathrm{HCl}$ ( $\mathrm{pH} \mathrm{8.3),} 50 \mathrm{~mm}$ $\mathrm{KCl}, 1.5 \mathrm{mM} \mathrm{MgCl}_{2}$; dNTPs at $200 \mu \mathrm{M}$; primers at $0.25 \mu \mathrm{M}$; and Taq polymerase at $2.5 \mathrm{U} / 100 \mu \mathrm{l}$. The cycle parameters were $55^{\circ} \mathrm{C}$ for $1 \mathrm{~min}, 72^{\circ} \mathrm{C}$ for $1.5 \mathrm{~min}$, and $95^{\circ} \mathrm{C}$ for $1 \mathrm{~min}$, with a final extension of 5 min at $72^{\circ} \mathrm{C}$ after 40 cycles.

\section{Preparation of Target}

The target fragment was $416 \mathrm{bp}$ in size and could be prepared from plasmid by amplification using the primer set designated $\mathrm{H} 2$ and $\mathrm{H} 4$. (These primers were common to all competitors used in this study.) The product was resolved by gel electrophoresis, isolated by the freeze- squeeze technique, ${ }^{(8)}$ quantitated by UV spectroscopy, and stored in a serial dilution for subsequent evaluation of the competitors.

\section{Construction of Competitors}

\section{Intron Competitor (980C)}

Genomic DNA was extracted from genital skin fibroblasts using the procedure of John et al. ${ }^{(9)}$ Intron competitor $980 \mathrm{C}$ was obtained as a single product from a PCR using primers $\mathrm{H} 2$ and $\mathrm{H} 4$ and genomic DNA as template. This competitor had an estimated size of 980 bp determined by comparison with molecular weight markers. The intron occurred at location 122 of the 416 -bp target sequence.

\section{Mutant Competitor (416C)}

The 416-bp target contains a PvuI site (CGAT/CG), $35 \mathrm{bp}$ from the 5' end, which can be converted to an NcoI site (C/CATGG) in the competitor by a 2-base change. This was achieved by substituting the $\mathrm{H} 4$ primer with a 47 -mer primer constructed with a 19-base extension to the $\mathrm{H} 4$ primer. The mutant product obtained from PCR using the standard conditions was isolated by freeze-squeeze after agarose gel electrophoresis and subcloned into the pGem-T vector. In addition to purifying the product, this procedure provides the option of generating a competitor at the mRNA level. Amplifying the subcloned plasmid with primers $\mathrm{H} 2$ and $\mathrm{H} 4$ gave the required product, which was serially diluted as described for the target.

\section{Deletion-type Competitors}

\section{6-bp Competitor (336C)}

The 416-bp target contained one MspI site at location 193. Using plasmid as template, a 128-bp fragment was generated with primer $\mathrm{H} 4$ and a mutagenic primer that created a new MspI site at location 113 . The 416- and 128-bp products were digested with MspI, and fragments of 223 and 113 bp were purified with each containing a MspI digestion end and complimentary coding to the primers $\mathrm{H} 2$ and $\mathrm{H} 4$, respectively. These products (50 ng each) were ligated in 10 $\mu l$ of buffer [66 mM Tris- $\mathrm{HCl}, 5 \mathrm{~mm}$ $\mathrm{MgCl}_{2}, 1 \mathrm{~mm}$ DTT, and $1 \mathrm{~mm}$ ATP $(\mathrm{pH}$ 7.5)] with 3 units of T4 DNA ligase for 2 hr at room temperature. A $1-\mu l$ portion of this mixture was amplified by PCR with primers $\mathrm{H} 2$ and $\mathrm{H} 4$. The reaction product was electrophoresed, and the 336-bp band was excised, subcloned, and quantified as described above. (It was found necessary to purify this competitor and all others that involved a ligation step in their construction by subcloning into a pGem-T vector. This prevented the formation of any nonspecific products when amplifying from very high dilutions.)

\section{2-bp Competitor (272C)}

The 416-bp target contained an MspI restriction site at location 193 and an SphI site at location 49 . The target was digested with each of these enzymes, and the 49- and 223-bp fragments were isolated as above. The restriction sites were treated with Klenow DNA polymerase (reaction mixture: $50 \mathrm{ng}$ of products, 5 units of Klenow, $1 \times$ PCR buffer, $1 \mathrm{~mm}$ dNTPs in $10 \mu \mathrm{l}$ ) at room temperature for $30 \mathrm{~min}$, ligated, purified, and quantified as for $336 \mathrm{C}$.

\section{Nonhomologous Competitors}

$600 \mathrm{C}$

This competitor was prepared by a modification of the method of Siebert et al. ${ }^{(5)}$ The nonhomologous insertion fragment was obtained from a downstream segment of the AR plasmid, which had a similar $G+C$ content to the target sequence. Digestion of this segment with $N c o I$ and HindIII gave a 551-bp fragment with a 4-base 5 ' overhang at each site. This fragment was ligated to the $\mathrm{H} 2$ and H4 primer-primer compliments with the reciprocal overhangs. The ligation reaction $(20 \mu \mathrm{l})$, which contained 1 pmole of each primer, primer compliment, and insert and 3 units of DNA ligase, was performed at $16^{\circ} \mathrm{C}$ for $1 \mathrm{hr}$. After heating to $75^{\circ} \mathrm{C}$ for $5 \mathrm{~min}, 1 \mu \mathrm{l}$ of ligation product was amplified with primers $\mathrm{H} 2$ and $\mathrm{H} 4$ using standard conditions for 30 cycles. The 600 -bp product was isolated by the freeze-squeeze technique and subcloned as above.

\section{$363 C$}

This competitor was prepared to provide a standard that closely matched the target (416 bp). The insert for ligation was 
constructed from the same downstream segment as above except that a new HindIII site was generated with a mutant primer that yielded a 311-bp fragment with the required $5^{\prime}$ overhangs after digestion. This insert was treated as above to yield a 363-bp competitor.

\section{Test for Heteroduplex Formation}

The following approach was used to determine whether the competitor was susceptible to heteroduplex formation with the target. An equal mixture of the two components was prepared at concentrations that could be readily visualized by agarose gel electrophoresis $(60 \mathrm{ng} / \mu \mathrm{l})$, and half was heated to $95^{\circ} \mathrm{C}$ for $5 \mathrm{~min}$. The treated and untreated mixtures were electrophoresed on $2 \%$ agarose gel, and the presence or absence of extra bands noted. If only two bands were detected after heating, the ratio of band intensities was measured, as heteroduplexes may form that cannot be resolved from a homoduplex. Under these circumstances, one of the bands will increase in intensity while the other decreases.

\section{Test for Differences in Amplification Efficiences}

To establish whether amplification efficiencies were equal, a mixture of equal concentrations of competitor and target $(60 \mathrm{ng} / \mu \mathrm{l})$ was diluted 1 in $10^{6}$ to the working level of the assay and $1 \mu \mathrm{l}$ was reamplified for 40 cycles. The ratio of competitor to target was determined before and after amplification by laser densitometry (Bio-Rad model GS-670 imaging densitometer). A change in the ratio after amplification provided a direct measure of amplification efficiency in the absence of heteroduplex formation. If resolvable heteroduplexes were detected, their relative contribution was determined and apportioned equally between target and competitor. If the ratio of components had changed, the difference in amplification efficiencies was confirmed by one of the following procedures.

\section{Titration}

A series of tubes, each containing $60 \mathrm{fg}$ of target and a range of competitor concentrations, were amplified using standard conditions for 40 cycles and the equivalence point was determined. (The equivalence point is defined as the point at which the target and competitor produce bands of equal intensity following electrophoresis.) The initial ratio of target to competitor at equivalence reflected directly the amplification differences over 40 cycles. This method was suitable for detecting twofold or greater differences in efficiencies over 40 cycles.

\section{Radionucleotides}

The same amplification approach described above was performed in the presence of $0.2 \mu \mathrm{Ci}$ of $\left[{ }^{32} \mathrm{P}\right] \mathrm{dCTP}$. The products were separated on $2 \%$ agarose gel, the bands were excised, and radioactivity was determined by scintillation counting. When resolvable heteroduplexes were present these bands were also excised and their counts equally apportioned to target and competitor. The ratio of counts of target to competitor was determined and used to calculate efficiencies directly.

\section{Preparation of CDNA}

Total RNA was extracted from genital skin fibroblasts by the method of Chomczynski et al. ${ }^{(10)}$ The concentration of each specimen was determined by UV spectroscopy, and the samples were diluted with water to $1 \mu \mathrm{g} / \mu \mathrm{l}$. Reverse transcription was performed using $1 \mu \mathrm{g}$ of total RNA in $20 \mu$ l of buffer $[10 \mathrm{~mm}$ Tris$\mathrm{HCl}(\mathrm{pH} \mathrm{8.8)}$ ] containing $50 \mathrm{mM} \mathrm{KCl}, 1$ mM dNTPs, $5 \mathrm{~mm} \mathrm{MgCl}_{2}$, 15 units of $\mathrm{AMV}$, and $12.5 \mu \mathrm{M}$ primer $\mathrm{H} 2$ at $42^{\circ} \mathrm{C}$ for $20 \mathrm{~min}$. The reaction mixture was heated to $95^{\circ} \mathrm{C}$ for $5 \mathrm{~min}$ and stored at $-70^{\circ} \mathrm{C}$ until analysis. All samples were transcribed in duplicate.

\section{Determination of CDNA}

The titration procedure for all competitors was similar and was based on the procedure of Fandrey and Bunn. ${ }^{(6)}$ A volume of $1 \mu \mathrm{l}$ of reverse transcriptase product (equivalent to $50 \mathrm{ng}$ total RNA) was placed into each of a series of tubes containing a range of concentrations of competitor, and PCR was performed in a $50-\mu l$ volume using standard conditions. Generally, a twofold serial dilution was used except for the mutant competitor in double-cut mode in which the greater sensitivity of the equivalence point enabled twice the degree of discrimination. When using the mutant competitor, 20 $\mu l$ of the PCR product was digested with either PvuI (single cut) or individually with PvuI and NcoI (double cut) by the addition of 0.5 units of enzyme for $16 \mathrm{hr}$ at $37^{\circ} \mathrm{C}$. PCR products were run on $2 \%$ agarose gel containing ethidium bromide and photographed; and the equivalence point was determined by visual inspection. If the equivalence point coincided with a tested concentration of competitor, that value was used; if it fell between two concentrations, the average of these became the equivalence point. All determinations were performed in duplicate.

\section{RESULTS}

Six competitors were prepared using modifications of previously published methods. The primers and plasmid details used in the constructions are illustrated in Figure 1. Each competitor was synthesized to be amplified by a common primer set, $\mathrm{H} 2$ and $\mathrm{H} 4$, to facilitate a comparison of their respective efficiencies. This primer set had been used pre-

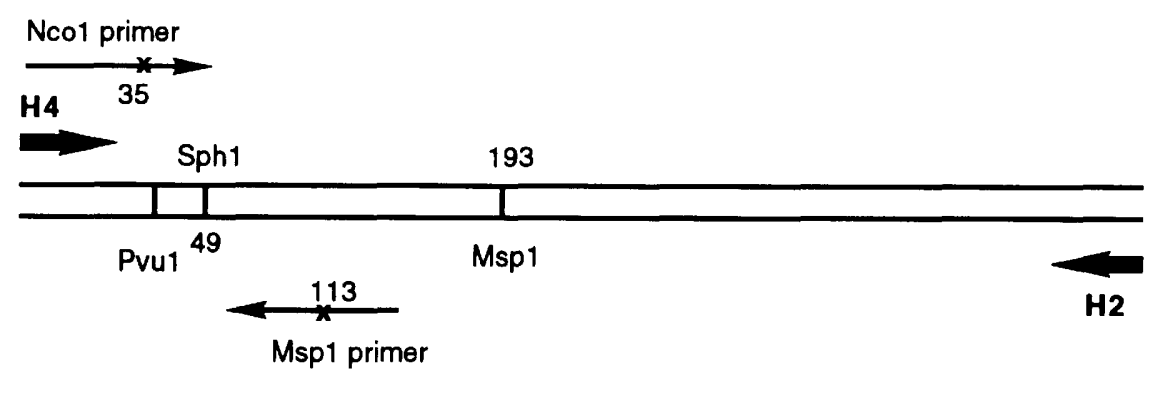

FIGURE 1 Target segment of CMVhAR3.1 plasmid showing relevant restriction sites and primers used in competitor constructions. 
viously in sequencing androgen receptor mRNA and was specific for the target. The antisense primer $\mathrm{H} 2$ was also used for the reverse transcription of mRNA. In this study mRNA was determined at the level of cDNA. Results are expressed as femtograms of cDNA per microgram of total RNA. Although cDNA levels have been obtained for 11 subjects, most of the comparisons were performed on a subset of four subjects comprising two patients and two controls. A summary of data relating to the synthesis, properties, and performances of the six competitors is presented in Table 1. Evidence for the formation of heteroduplexes among the target and the different sized competitors and for different amplification efficiencies among target and competitors is summarized in Figure 2. Each competitor is reported in further detail below.

\section{Deletion Construct Competitors}

Synthesis

In the published deletion construct procedure $^{(2)}$ the target contains a pair of restriction sites through which a segment of bases is eliminated to generate a competitor. As our target did not contain two suitable sites, we used modified procedures to prepare the two deletion type constructs $272 \mathrm{C}$ and $336 \mathrm{C}$.

\section{Heteroduplex Formation}

When amplified mixtures of $272 \mathrm{C}$ and target were separated on a $1 \%$ agarose gel, only two bands were observed. However, when the same product was run on a $2 \%$ agarose gel, the slower running band resolved into two distinct products suggesting heteroduplex formation (Fig. $2 \mathrm{a}$, lane 3 ). When the same procedure was repeated using $336 \mathrm{C}$ and target, there was slight broadening of the top band on $2 \%$ agarose but complete separation was not achieved. However, heteroduplex formation was established for both competitors in the procedure in which an equal mixture of each competitor and target was heated to $95^{\circ} \mathrm{C}$ for 5 min. The change in band ratios from 0.88 to 0.60 following heating of $336 \mathrm{C}$ with target would suggest the formation of a heteroduplex that is being electrophoresed at almost the same rate as the target (Fig. 2b, lanes 1,2).

\section{Amplification Efficiences}

\section{$272 C$}

Using data obtained from the densiometric analysis of Figure $2 \mathrm{a}$, the competitor was found to amplify with an efficiency of 2.4 times the target over 40 cycles. Titration experiments yielded a twofold difference in efficiencies. This equates to an $\sim 2.5 \%$ difference in efficiency per cycle.

\section{$336 C$}

The occurrence of heteroduplexes that

TABLE 1 Summary of the Construction, Characteristics, and Performance of Six Competitors Used

\begin{tabular}{|c|c|c|c|c|}
\hline $\begin{array}{l}\text { Competitor } \\
\text { type }\end{array}$ & $\begin{array}{l}\text { Size } \\
\text { (bp) }\end{array}$ & $\begin{array}{l}\text { Construction } \\
\text { steps }\end{array}$ & $\begin{array}{l}\text { Heteroduplex } \\
\text { formation }\end{array}$ & $\begin{array}{l}\text { Amplification } \\
\text { efficiency }\end{array}$ \\
\hline Mutant & 416 & 1 PCR with mutant primer & yes & 1 \\
\hline \multirow[t]{4}{*}{ Deletion constructs } & 272 & $\begin{array}{l}\text { digestions } \\
\text { ligation }\end{array}$ & & \\
\hline & & PCR & yes & 2.42 \\
\hline & 336 & $\begin{array}{l}\text { digestions } \\
\text { ligation }\end{array}$ & & \\
\hline & & PCR & yes & 1.18 \\
\hline Intron & 980 & 1 PCR with genomic DNA & yes & 0.14 \\
\hline \multirow[t]{4}{*}{ Nonhomologous } & 600 & $\begin{array}{l}\text { digestion } \\
\text { ligation }\end{array}$ & & \\
\hline & & PCR & no & 0.59 \\
\hline & 363 & $\begin{array}{l}\text { digestion } \\
\text { ligation }\end{array}$ & & \\
\hline & & PCR & no & 1.02 \\
\hline
\end{tabular}

${ }^{a}$ All competitors except intron were purified by subcloning into pGem-T vectors.

${ }^{b}$ Ratio of competitor to target as determined by densitometry after amplification of equal amounts for 40 PCR cycles.

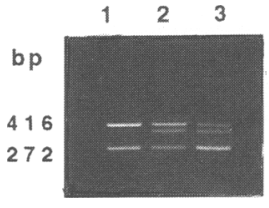

(a)

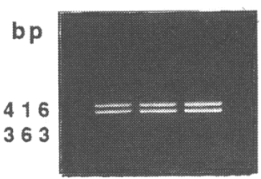

(c) (b)

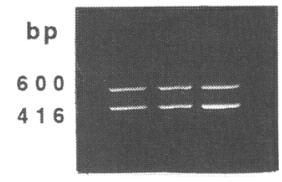

(d)
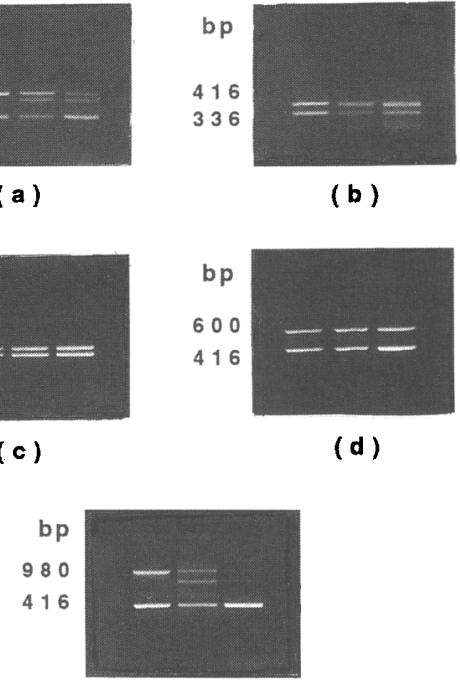

(e)

FIGURE 2 Tests for heteroduplex formation and differences in amplification efficiencies among the target and different sized competitors. Equal mixtures of competitor and target at $60 \mathrm{ng} / \mu \mathrm{l}$ (lane 1 ) were heated to $95^{\circ} \mathrm{C}$ for 5 min to establish susceptibility to heteroduplex formation (lane 2) and diluted 1 in $10^{6}$ and reamplified for 40 cycles to determine differences in amplification efficiency (lane 3 ). Band intensities were determined by densitometry and used to calculate relative amplification efficiencies (see Table 1). Results are shown for the competitors (a) 272C; (b) 336C; (c) 363C; (d) 600C; (e) 980C.

could not be resolved from target made this determination more difficult. An equal mixture of 336C and target at 60 ng/ $\mu \mathrm{l}$ was diluted 1 in $10^{6}$, amplified for 40 cycles, and heated to $95^{\circ} \mathrm{C}$ for $5 \mathrm{~min}$ to maximize heteroduplex formation. The ratio of $336 \mathrm{C}$ to the slower running band was 0.71 (Fig. 2b, lane 3). This represented the summation of two effects, heteroduplex formation and amplification efficiencies. To obtain the comparative amplification rate of $336 \mathrm{C}$ to target, the ratio of the amplified mixture was compared with the ratio of the heated mixture of 0.60 (Fig. 2b, lane 2) rather than the initial mixture, to isolate the effect of heteroduplex formation. This resulted in an amplification rate for $336 \mathrm{C}$ of 1.18 times that of the target.

\section{Nonhomologous Competitors}

Synthesis

Each competitor was prepared by ligating nonhomologous inserts obtained 
from a downstream region of the androgen receptor plasmid with a mixture of the $\mathrm{H} 2$ and $\mathrm{H} 4$ primers. Their compliments contained the appropriate 5 ' overhangs.

\section{Heteroduplex Formation and Amplification Efficiencies}

When either $363 \mathrm{C}$ or $600 \mathrm{C}$ was heated with the 416-bp target at $95^{\circ} \mathrm{C}$ for $5 \mathrm{~min}$, there was no evidence of heteroduplex formation (see Fig. 2c,d, lane 2). Data obtained from densitometric readings of the gels shown in Figures 2, $\mathrm{c}$ and d, gave amplification efficiencies relative to target over 40 cycles of 1.02 and 0.59 for $363 \mathrm{C}$ and $600 \mathrm{C}$, respectively. When $\left[{ }^{32} \mathrm{P}\right] \mathrm{dCTP}$ was used to quantitate the bands, the respective values were 1.07 and 0.52 .

\section{The Intron Competitor (980C)}

This was constructed from genomic DNA using the primers $\mathrm{H} 2$ and $\mathrm{H} 4$. The size of the product was estimated to be $980 \mathrm{bp}$ using molecular weight markers. It was not further purified.

\section{Heteroduplex Formation and Amplification Efficiency}

When mixtures of $980 \mathrm{C}$ and target (each at $60 \mathrm{ng} / \mu \mathrm{l}$ ) were heated using the same conditions described for the deletion constructs, an extra two bands appeared on a $2 \%$ agarose gel (see Fig. 2e). Coincidently, the intensity of the two homoduplexes was reduced. When the same mixture of $980 \mathrm{C}$ and target used in the above experiment was diluted 1 in $10^{6}$ and reamplified over 40 cycles the 416 bp band predominated over the intron product when visualized on agarose gel suggesting a large difference in amplification efficiencies. Using densitometry and adjusting for heteroduplex formation the intron competitor was calculated to amplify at $\sim 0.14$ of the rate of the target. By titration it was found that a 1:6 ratio of target to $980 \mathrm{C}$ gave equal products over 40 cycles, that is, the target was amplified six times more efficiently than the competitor. Because of the large difference in amplification efficiency the intron competitor was not used in cDNA determinations.

\section{Mutant Competitor}

\section{Amplification Efficiency}

An equal amount of target and mutant (60 fg) was amplified for 40 cycles, and the PCR product was divided and digested individually with PvuI and NcoI. When the digestions were electrophoresed on $2 \%$ agarose gel, the amount of cut and uncut from each enzyme was the same confirming that both target and mutant amplified with the same efficiency.

\section{Heteroduplex Formation}

Because the target and competitor share $>99 \%$ of common sequence then, under annealing conditions, their singlestranded DNAs will hybridize with the compliment of one another with almost the same affinity as they would to their own. This will produce heteroduplexes as illustrated in Figure 3. If the target and competitor are initially in a ratio of a: 1 then following strand separation and reannealing the four duplexes in Figure 3, based on probability of collision, should occur in the ratio of $\mathrm{a}^{2}: \mathrm{a}: \mathrm{a}: 1$. As the heteroduplexes are resistant to digestion by either enzyme, digestion of the mixture with $P v u$ I will result in $a^{2} / a^{2}+2 a+1$ of the product being cut while digestion with $N$ coI will lead to $1 / \mathrm{a}^{2}+2 \mathrm{a}+1$ of it being cut. In our evaluation of a singlecut mutant competitor assay we used PvuI to digest the products. At the equivalence point, defined as when cut $=$ uncut, and when heteroduplex formation is maximal, then $a^{2}=2 a+1$, which solves for $a=2.414$. With no heteroduplex formation, $\mathrm{a}=1$. When calculating the concentration of target, the value a must be factored into the result, that is, the degree of heteroduplex formation must be known.
For the mutant competitor assay being used in the double-cut mode the equivalence point is defined as when the ratio of cut/uncut using $P v u I$ to digest the mixture equals the ratio of cut/uncut using Ncol. Then, at maximal heteroduplex formation, $a^{2} / 2 a+1=1 / a^{2}+2 a$ solving for $a=1$. Therefore, the equivalence point using the double-cut strategy should always occur when there are equal amounts of target and competitor, independent of the degree of heteroduplex formation. Only the ratio of cut/uncut should vary at equivalence ranging from $1: 3$ at maximal heteroduplex to $1: 1$ with none.

To simplify analysis we used a high cycle number in the amplification to maximize heteroduplex formation., For the four subjects in which cDNA levels were determined, each equivalence point using the double-cut strategy was between two to three times that using a single cut, consistent with the above considerations. Results from a typical subject are shown in Figure 4 . The average values for the equivalence points were $1440 \mathrm{fg}$ of $\mathrm{cDNA} / \mu \mathrm{g}$ of total RNA for the double cut and $640 \mathrm{fg} \mathrm{cDNA} / \mu \mathrm{g}$ total RNA $(n=4)$ for the single cut. Provided the PCR is terminated in the plateau phase, a valid determination should be achieved when using a single cut by multiplying the observed equivalence point by 2.4 .

\section{Heteroduplex Formation and Cycle Number}

The degree of heteroduplex formation was found to be cycle dependent. cDNA values for three subjects were determined at alternate cycle numbers beginning when product could be first visualized on agarose gel (usually cycle 28) through to the plateau stage (cycle 36). All samples were cut with both PvuI and NcoI. In each case, the equivalence point

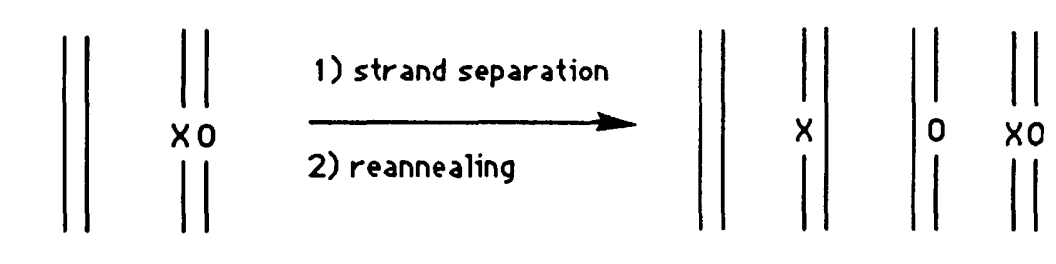

\section{target competitor \\ (PVU1) (NCO1)}

\section{RATIO O : 1}

$$
\mathbf{8}^{2}: \mathbf{0}: \mathbf{a}: \mathbf{1}
$$

FIGURE 3 Schematic representation showing heteroduplex formation and the ratio of possible products. 

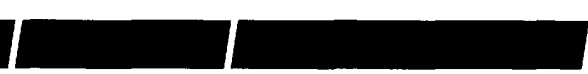

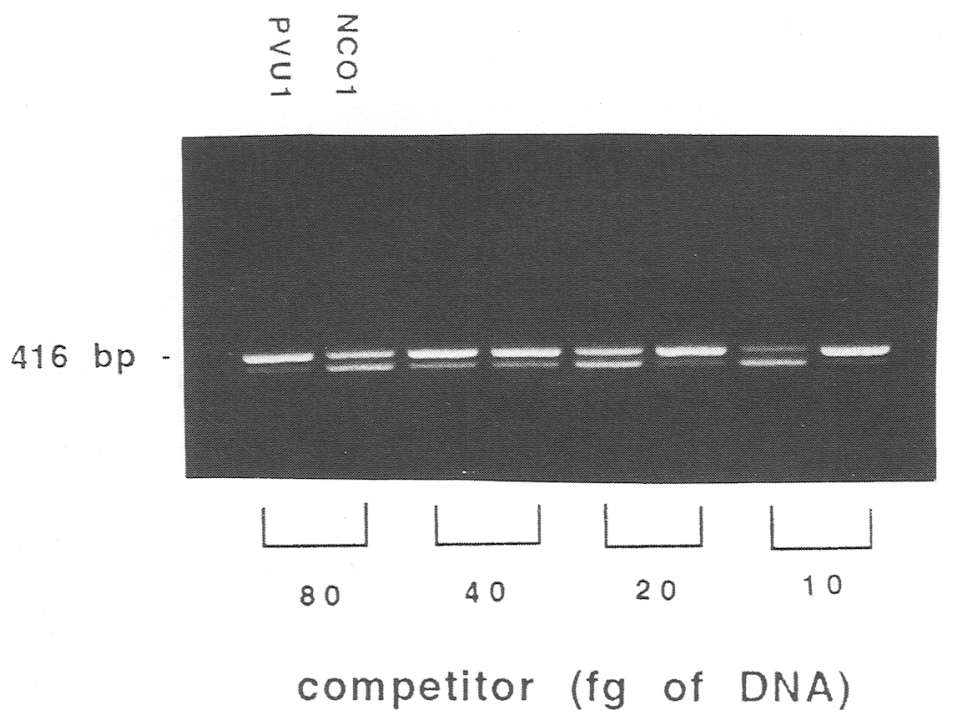

FIGURE 4 Determination of androgen receptor mRNA (as cDNA) from 50 ng of total RNA using a mutant competitor in the double-cut mode. For each pair of competitor concentrations, the products from $P v u \mathrm{I}$ digestion (left) and from $\mathrm{Ncol}$ (right) are shown. The equivalence point is 40 $\mathrm{fg} / 50 \mathrm{ng}$ of RNA. From the same information the equivalence point using $P v u \mathrm{I}$ in the single-cut mode can be determined and is almost $20 \mathrm{fg} / 50 \mathrm{ng}$ of RNA. This is in agreement with the theoretical prediction that the actual value obtained using a mutant competitor with a single cut can be achieved by multiplying the observed equivalence point by 2.4 , assuming maximal heteroduplex formation. as defined for the double-cut method was constant and independent of cycle number, whereas the equivalence point using $P v u \mathrm{I}$ as a single cut decreased by a factor of $\sim 2$ (representing one or two serial dilutions) as the PCR went from the shown).

The most likely explanation for these observations is that during the linear phase, primers rather than product anneal to the template, which following extension will result in homoduplex formation. At plateau product mixture anneals with itself in preference to primers eroduplex formation. Providing the amplification is terminated in either the linear or plateau stage, the concentration of target can be determined when using a single cut. However, during the transition stage when heteroduplex formation is submaximal, analysis is very complex. When using the double-cut method we have found the determinations to be independent of cycle number.

\section{Relationship between Amplification Efficiency and Size}

When the relative amplification rate of each competitor was plotted against competitor size a significant inverse exlinear to the plateau phase (data not resulting in both homoduplex and het- ponential relationship was observed (see Fig. 5).

\section{Standardization of Competitors vs. Target}

Generally, in PCR assays, levels of mRNA/cDNA are expressed in terms of the amount of competitor added at the equivalence point, which assumes equal amplification efficiences and no unresolvable heteroduplexes. We found that it is still possible to use a competitor if these conditions are not met providing the competitor is first standardized against the target. To achieve this, the 416-bp target at a concentration of 200 $\mathrm{fg} / \mu \mathrm{l}$, which was at the top end of our working range, was titrated against a series of dilutions of the competitor using standard conditions of amplification. The concentration of competitor that gave an equal product with the target after amplification and visualization was assigned the relative concentration of $200 \mathrm{fg} / \mu \mathrm{l}$. This solution of competitor was used to provide a series of dilutions of known relative concentrations across the working range of the assay. When these standardized competitors were used to determine the cDNA levels in our subjects, the results were in good agreement with values obtained using the mutant competitor in the double-cut mode. Comparative results using standardized and nonstandardized competitors for a typical subject are shown in Table 2.

\section{DISCUSSION}

The initial aim of this study was to establish a simple and reliable method for measuring levels of mRNA of the androgen receptor. In evaluating some methods published recently, we have pro-

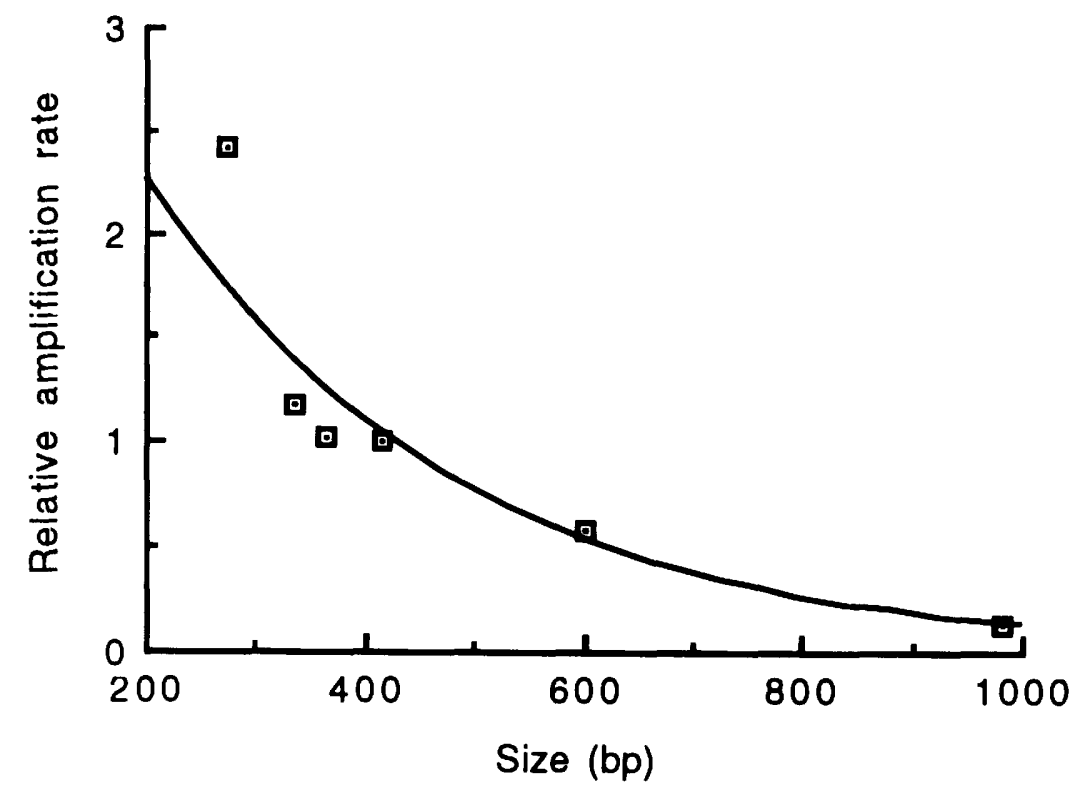

FIGURE 5 Plot of ratio of amplification rates of competitors relative to 416-bp target against competitor size (bp) using an exponential curve fit. The amplification rates are from Table 1. Data fit the relationship $y=4.64 \times 10(-1.56 e-3 x)$, with $R^{2}=0.96$. 
TABLE 2 Equivalence Points Determined for a Control Subject Using Standardized and Nonstandardized Competitor Dilutions

\begin{tabular}{lcc}
\hline & \multicolumn{2}{c}{ Equivalence point (fg of cDNA) } \\
\cline { 2 - 3 } Competitor type & without standardization & with standardization \\
\hline Mutant & 40 & $96^{\mathrm{a}}$ \\
$\quad$ single cut $(P v u \mathrm{I})$ & 100 & not applicable \\
$\quad$ double cut & 40 & 100 \\
Deletion constructs & 160 & 100 \\
272C & 120 & 120 \\
336C & 240 & 120 \\
Nonhomologous & & \\
363C & & \\
600C & & \\
\hline
\end{tabular}

$20,40,60,80,120,160,240$, or $320 \mathrm{fg}$ of competitor ( \pm standardization) was added to tubes containing target cDNA transcribed from $50 \mathrm{ng}$ of total RNA and amplified for 40 cycles. Results are expressed as the amount of competitor at equivalence.

aDerived by multiplying equivalence point by 2.4 .

duced several findings that may be of importance when planning to use competitive PCR to determine mRNA levels.

First, the use of a common primer set to amplify DNAs of different size does not guarantee equal amplification efficiences. For the six competitors constructed for this study, there was $\sim 12$ fold difference in relative product yield between the least and most efficient competitors over 40 PCR cycles. In some previous studies equal efficiencies have been demonstrated over a relatively small number of cycles. Differences of $1 \%$ or $2 \%$ in efficiency per cycle, which might not be obvious over 5 or 10 cycles, can translate into up to a twofold difference in product yield in a typical PCR. When measuring a rare species of mRNA, which requires greater amplification, any small differences in efficiencies need to be minimized. To test for differences in amplification efficiencies an equal mixture of target and competitor that has been previously visualized on agarose can be diluted to the working level of the assay and reamplified using standardized conditions. Any change in product ratios from the original mixture reflect differences in amplification efficiency.

This approach minimizes possible errors arising from dilutions and spectroscopy. The observed difference can be confirmed by titration experiments or by the use of radionucleotides if the differences are small. With the competitors used in this study we observed an inverse exponential relationship between amplification efficiency and template size. From these results we would suggest that when constructing a competitor it is important to minimize size difference with the target. In a recent review, Clementi and co-workers also emphasized the need to minimize differences when constructing deletion or insertion type competitors. ${ }^{(11)}$

A second finding of this study is that intron and deletion type constructs may still be susceptible to heteroduplex formation following amplification with target. That these formations could occur might reasonably be expected if it is considered that such constructs share major segments of sequence homology with the target. In this study heteroduplex formation increased as the PCR approached the plateau phase. Provided the heteroduplex can be resolved from the homoduplexes analysis is still straightforward. Recently, Piatak et al. ${ }^{(12)}$ have also reported observing heteroduplex formation when using a deletion construct in a competitive PCR assay in contrast to a number of previous reports in which, under similar circumstances, heteroduplexes were not observed. Problems arising from unresolvable heteroduplexes can be avoided by using a radionucleotide in the PCR. Because of the increased sensitivity of detection using this method, the PCR can be terminated at an earlier cycle number before heteroduplexes have begun to form.

In many reports mRNA or cDNA is expressed in terms of the absolute amount of competitor added, which assumes equal amplification efficiencies. Our study shows this is not always true and that differences in amplification efficiencies will have a direct effect on the absolute values being measured although relative differences between samples will not be significantly affected. The procedure of standardizing competitors against the target when such differences do occur should enable valid determinations to be made.

A potential disadvantage of using a mutant competitor is that the analysis can be dependent on the degree of heteroduplex formation. Whereas this may be true when the assay is used with a single cut, we present a theoretical basis and provide experimental evidence to show that the double-cut option of Fandry et al. ${ }^{(6)}$ produces determinations that are independent of heteroduplex formation and, therefore, cycle number. Furthermore, we have found that the equivalence point for this method, which is determined differently from the other options evaluated in this study, provides greater sensitivity. This is the simplest competitor to construct, with the exception of the intron competitor, which was not considered to be of practical value. Our modification, which used an extended primer to convert one restriction site to another, should have general applicability. We found that a minimum of a $10 \%$ difference in size between target and competitor was necessary to achieve reasonable separation on a $2 \%$ agarose gel. Thus, this modification is practical for constructing mutant competitors for use with target fragments up to $500 \mathrm{bp}$.

When we compared the overall usefulness of the different competitors the mutant competitor used in the doublecut mode was technically the most reliable method. However, by nature of its design, more sample handling is required. An excellent alternative for our purposes was to use a nonhomologous competitor of similar size to the target. In summary, we found competitve PCR to be a most reliable and straightforward technique with the qualification that when absolute levels of mRNA are being determined the competitor used should be assessed for its ability to amplify with the same efficiency as the target and for its capacity to form heteroduplexes with it.

\section{ACKNOWLEDGMENTS}

We thank the Royal Perth Hospital Medical Research Foundation for their support. 


\section{REFERENCES}

1. Galea, E. and Feinstein, D.L. 1992. Rapid synthesis of DNA deletion constructs for mRNA quantitation: Analysis of astrocyte mRNAs. PCR Methods Applic. 2: 66-69.

2. Gilliland, G., S. Perrin, K. Blanchard, and F.H. Bunn. 1990. Analysis of cytokine mRNA and DNA: Detection and quantitation by polymerase chain reaction. Proc. Natl. Acad. Sci. 87: 2725-2729.

3. Wang, A.M, M.V. Doyle, and D.F. Mark. 1989. Quantitation of mRNA by the polymerase chain reaction. Proc. Natl. Acad. Sci. 86: 9717-9721.

4. Li, B., P. Sehajpal, A. Khanna, H. Vlassara, A. Cerami, K.H. Stenzel, and M. Suthanthiran. 1991. Differential regulation of transforming growth factor $\beta$ and interleukin-2 genes in human $T$ cells: Demonstration by usage of novel competitor DNA constructs in quantitative polymerase chain reaction. J. Exp. Med. 174: 1259-1262.

5. Siebert, P.D. and J.W. Larrick. 1993. PCR mimics: Competitive DNA fragments for use as internal standards in quantitative PCR. BioTechniques 14: 244-249.

6. Fandrey, J. and H.F. Bunn. 1993. In vivo and in vitro regulation of erythropoietin mRNA: Measurement by competitive polymerase chain reaction. Blood 81: $617-623$.

7. Tilley, W.D., M. Marcelli, J.D. Wilson, and M.J. McPhaul. 1989. Characterization and expression of a cDNA encoding the human androgen receptor. Proc. Natl. Acad. Sci. 86: 327-331.

8. Sarkar, G. and S.S. Sommer. 1990. The "Megaprimer" method of site-directed mutagenesis. BioTechniques 8: 404-407.

9. John, S.W., G. Weitzner, R. Rozen, and C.R. Scriver. 1991. A rapid procedure for extracting genomic DNA from leukocytes. Nucleic Acids Res. 19: 408.

10. Chomczynski, P. and N. Sacchi. 1987. Single-step method of RNA isolation by acid guanidinium thiocyanate-phenol-chloroform extraction. Anal. Biochem. 162: 156159.

11. Clementi, M., S. Menzo, P. Bagnarelli, A. Manzin, A. Valenza, and P. Varaldo. 1993. Quantitative PCR and RT-PCR in virology. PCR Methods Applic. 2: 191-196.

12. Piatak, M., K.C. Luk, W. Williams, and J.D. Lifson. 1993. Quantitative competitive polymerase chain reaction for accurate quantitation of HIV DNA and RNA species. BioTechniques 14: 70-81.

Received August 29, 1994; accepted in revised form December 2, 1994. 


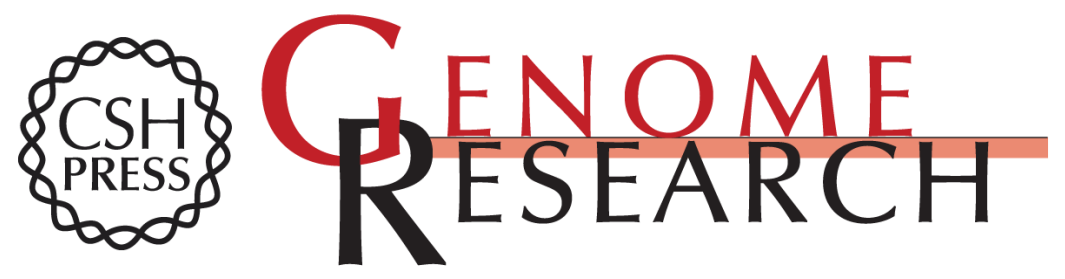

\section{An evaluation of competitor type and size for use in the determination of mRNA by competitive PCR.}

R K McCulloch, C S Choong and D M Hurley

Genome Res. 1995 4:219-226

References This article cites 12 articles, 5 of which can be accessed free at: http://genome.cshlp.org/content/4/4/219.full.html\#ref-list-1

License

Email Alerting

Receive free email alerts when new articles cite this article - sign up in the box at the Service top right corner of the article or click here.

\section{Affordable, Accurate Sequencing.}

To subscribe to Genome Research go to: https://genome.cshlp.org/subscriptions 